\title{
WINDOW OF OPPORTUNITY: USING THE INTERRUPTION LAG TO MANAGE DISRUPTION IN COMPLEX TASKS
}

\author{
Sheryl L. Miller \\ Department of Psychology \\ George Mason University \\ Fairfax, Virginia
}

Interruption is a fundamental human problem. Past research has focused on how people resume an interrupted task after attending to some unrelated secondary task, ignoring interruptions that are an integral part of overall performance. In many settings, such as air traffic control or invehicle navigation, people must integrate the processing of the interruption itself and the resumption of the interrupted task. An experiment was conducted to investigate how people manage interruptions in a team decision-making task where interruptions are an integral part of overall performance. One strategy proposed for managing interruptions is the "rehearsal strategy." It was expected that people who rehearsed where they would resume the task would be able to overcome the disruption imposed by interruption. The results indicate that people instructed to use this strategy behaved differently than those who were not instructed. However, instruction did not improve performance on interrupted tasks and may even have been detrimental.

\section{INTRODUCTION}

Interruption of cognitive processing is pervasive, for example, a car phone ringing, a colleague entering the room to ask a question, intelligent agents in the form of office assistants, and instant messaging interfaces. Previous research shows that people who are interrupted often fail to return to their original task in a timely fashion, or neglect the original task altogether (O'Conaill \& Frohlich, 1995; Dismukes, Young, \& Sumwalt, 1998) resulting in stress and a decreased sense of well-being (Kirmeyer, 1988; Zijlstra, Roe, Leonora, \& Krediet, 1999). McFarlane (1987) says that this distraction is the result of a person forgetting what he or she was doing prior to an interruption, leading to increased time-ontask and decreased accuracy upon task resumption.

Researchers have found a variety of characteristics that make interruptions most disruptive. These include high interruption frequency (Spier, Valacich, \& Vessey, 1999; Zijlstra, et al., 1999), similarity between interruptions and interrupted tasks, (Gillie \& Broadbent, 1989; Cellier \& Eyrolle, 1992), task or interruption complexity (Gillie \& Broadbent, 1989; Cellier \& Eyrolle, 1992; Spier, et al., 1999; Zijlstra, et al., 1999), and the relatedness of interruptions to the interrupted task (Spier, et al., 1999; Cutrell, Czerwinski, \& Horvitz, 2000).

Past research has isolated these elements by focusing on interruptions that are trivial elements of ongoing performance. However, in many tasks, interruptions are common and anticipated, and overall performance is dependent on the successful integration of interruptions into on-going task performance. In these kinds of cases, the task is complex and interruptions are frequent, similar to, and related to the interrupted task. Thus, task resumption is only a portion of performance. Integration of the interruption is crucial to performance, too. In complex tasks, people must learn to balance the resumption of the interrupted task and processing of the interruption itself. The experiment presented here investigates these complex tasks using team decision-making as the context in which interruptions occur.

In addition to understanding the characteristics that make interruptions disruptive, it is important to understand how people manage interruptions. How people use their memories to manage interruptions has been a point of controversy in the literature. Gillie and Broadbent (1989) concluded that neither the length of the interruption nor the ability to control the point of the interruption improved task resumption. Thus, when given the opportunity, people were not able to improve their memory for where they left off in the interrupted task.

However, Altmann and Trafton (2002) suggested this finding was due to a lack of experimental control over the interruption and the imposition of other unexamined strategies. In fact, several strategies for resuming an interrupted task have been proposed in the literature. These include speeding up the primary task (Latorella, 1996; Zijlstra, et al., 1999), utilizing external memory aids (Cutrell, et al., 2000), and postponing the interruption (Zijlstra, et al., 1999). Altmann and Trafton (2002) propose that there is indeed a "window of 
opportunity" (p. 65) during an interruption in which people are able to rehearse the task resumption point. In this framework, an interruption has three main parts: an alert (e.g., the phone ringing), an event (e.g., picking up the phone), and a conclusion (e.g., hanging up the phone). A window of opportunity, or interruption lag (Altmann \& Trafton, 2002) occurs between the interruption alert (e.g., the phone ringing) and the interruption event (e.g., picking up the phone). In a team communication task, the analogous interruption might be an alert (e.g., receiving the message from a teammate), an event (e.g., reading the message), and a conclusion (e.g., dismissing the message). An experiment was designed to investigate the use of the interruption lag to improve memory for the task resumption point. It was hypothesized that people who use the interruption lag to rehearse where they leave off in the interrupted task will be better able to resume the interrupted task compared to those who use no such strategy. The experiment presented here compared task performance among people instructed in this strategy and those who received no such instruction.

\section{METHOD}

\section{Participants}

Twenty-four undergraduates volunteered to participate in exchange for course credit. Participants were assigned to one of two rehearsal strategy conditions - Instructed or Not Instructed. Each participant gave informed consent and was seated at a Macintosh computer. Following the pretest and two counterbalanced experimental scenarios, participants were debriefed.

\section{Pre-test}

Because individual memory differences might influence the use of the rehearsal strategy, the n-back task was administered as a pretest to assess working memory capacity. Numbers $0-9$ were presented on the computer screen one at a time (20 numbers in each trial). Participants assessed whether the number on the screen was the same or different as one of the previous numbers - the last number, 2 numbers back, or 3 numbers back. After reaching criteria of $95 \%$ on the 1-back practice task, each participant performed 3 trials each of the 2back and 3-back task. The proportion correct was averaged across all trials to produce an overall score.

\section{Experimental Scenarios}

Following written, verbal, and computer instruction, each participant was given a break and then performed two scenarios on the I-ARGUS system (for a description of the ARGUS environment see Schoelles \& Gray, 2001). I-ARGUS is a simulation of a team radar operator decision-making task. Participants worked individually to assess each aircraft that appeared on the radar scope and to make recommendations to a "leader" about what course of action to take. Although the team was simulated, participants were instructed that the computer simulation behaved like a real team.

In each of two scenarios, 64 aircraft were displayed one at a time on a geocentric radar display. When an aircraft appeared, the participant would "hook" it by clicking on it to display three pieces of aircraft data (range, speed, and altitude). Each piece of data was assessed by the participant in terms of its threat level on a $0-3$ scale (e.g., speed of 600 knots is highly threatening or 3). Overall threat for the aircraft was determined by adding together the threat level of all the data. This overall score was used to determine the course of action for each aircraft.

Occasionally, a piece of data (speed or altitude) was unavailable for a particular aircraft. In this case, participants were instructed to make the best decision that they could with missing data. However, sometimes the missing data was received in the form of a message from a teammate prior to the arrival of the relevant aircraft. These messages served as interruptions to aircraft decision-making.

Each interruption was composed of an interruption lag and the message itself (Figure 1). The interruption lag began when an interruption alert hid the radar screen from view. At this point, participants needed to select to read the message, ending the interruption lag. After selecting to read the message, the message data appeared giving the participant a piece of data about the current or a future aircraft. When participants dismissed this data, the interrupted aircraft reappeared on the screen and the message was no longer available. Therefore, participants needed to remember the message information while they resumed the interrupted decisionmaking and while they made decisions on subsequent aircraft. 


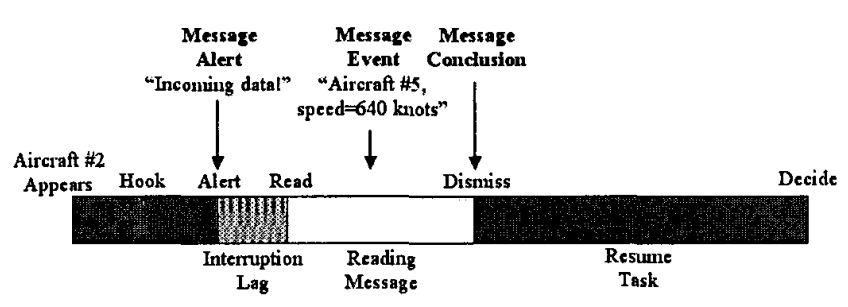

Figure 1. Anatomy of an interruption (based on Altmann \& Trafton, 2002). Aircraft \#2 exemplifies interrupted decision-making. The interrupting message is relevant to a future aircraft (\#5).

All participants were presented with 5 categories of aircraft, defined by the amount of data initially available and whether or not decision-making for that aircraft was interrupted (see Table 1). The first two types of aircraft served as baseline performance tasks. How well do people make decisions when they have complete data and when they have missing data? The next three types of aircraft were used to investigate the impact of interruptions on ongoing and future performance. Here, interruptions are messages that contain data that will not be needed immediately, but for some future aircraft. This creates a requirement for participants to remember a message while they resume the interrupted task and during the course of several future decisions.

\begin{tabular}{|c|c|c|c|}
\hline Aircraft & Initial Data & $\begin{array}{c}\text { Intemupted } \\
\text { Decision-making? }\end{array}$ & Description \\
\hline Complete data & Complete & No & baseline best \\
\hline Missing data & Missing & No & baseline worst \\
\hline Internupted & Complete & Yes & $\begin{array}{c}\text { ressage \& nust remember } \\
\text { it for a future aircraft }\end{array}$ \\
\hline $\begin{array}{c}\text { Remembering } \\
\text { message }\end{array}$ & Complete & No & $\begin{array}{c}\text { remembering an earlier } \\
\text { message for use on a } \\
\text { future aircraft }\end{array}$ \\
\hline $\begin{array}{c}\text { Utilizing } \\
\text { message }\end{array}$ & Missing & No & \begin{tabular}{c} 
utilize earlier message \\
\hline
\end{tabular}
\end{tabular}

Table 1. Types of aircraft presented to participants.
Note that a 6th category of aircraft, in which interruptions were immediately relevant to the resumed task, was also presented to participants. This category will not be discussed further due to the length restrictions of this paper.

Participants in the instructed condition were instructed to take advantage of the interruption lag to try to remember where they "left off" in the task using a rehearsal strategy. Non-instructed participants were given no strategy information. Task performance was assessed in terms of accuracy (proportion correct), total time to make a decision (subtracting out the time spent reading a message), and where appropriate, interruption lag time and time spent reading a message. Note that outliers greater than four standard errors from the mean were eliminated from the analyses. An analyses of planned comparisons was used to investigate the effects of interruption on performance (compared to baseline best performance). Additionally, the analyses addressed whether instruction in the rehearsal strategy mediates the effect of interruption.

\section{RESULTS}

Interruption had a significant effect on performance in terms of time (in seconds) to make a decision but not in terms of accuracy (proportion correct $M=0.76, S D=0.27, t(23)=-0.427, p=0.673)$. The total time to make a decision for interrupted aircraft was 20.73 seconds $(S D=5.37)$ which was significantly longer than the time to make a decision for non-interrupted aircraft $(M=14.65, S D=3.53)(t(23)=10.238, p<0.001)$. Interestingly, the time spent issuing a decision after an interruption $(M=13.61, S D=4.54)$ is the same as the time to make a non-interrupted decision after hooking the aircraft $(M=13.16, S D=3.37)(t(23)=0.958 . p=0.348)$. This implies that participants essentially "started over" following an interruption, even though they had spent approximately 6 seconds working on the decision (after hooking the aircraft) prior to reading the interrupting message.

Did instruction in the rehearsal strategy improve how long it took to make a decision for interrupted aircraft? There was a marginally significant difference for total decision time between instructed and noninstructed participants for interrupted aircraft $(t(22)=-$ $1.969, p=0.062$ ). However, this result was opposite the direction which was expected. Instructed participants took over 4 seconds longer to make decisions when they were interrupted $(M=22.77, S D=3.80)$ than participants who where not instructed $(M=18.70, S D=6.07)$. Instruction had no effect on accuracy for interrupted decision-making $(M=0.75, S D=0.26, t(22)=0.058$, 
$p=0.954)$. Additionally, working memory pretest scores were not correlated with these performance measures (all $p>0.160$ ).
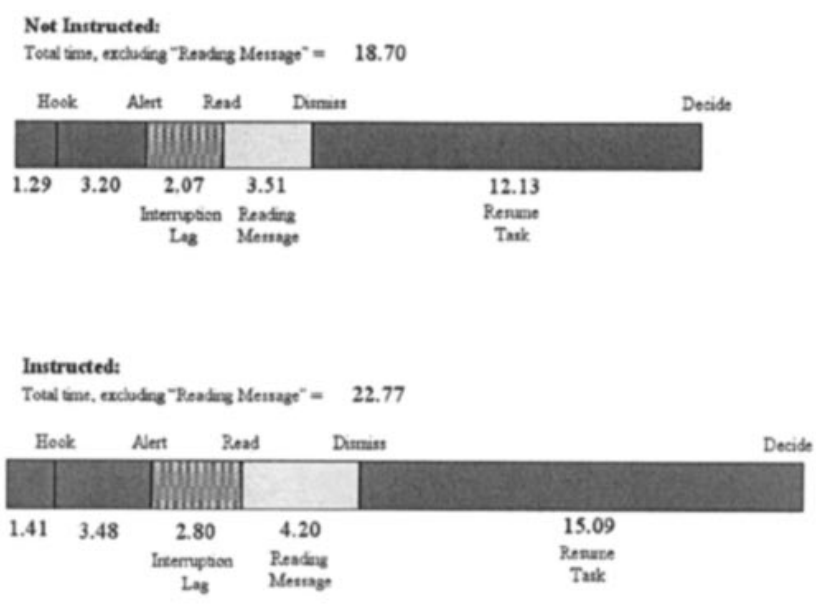

Figure 2. Response times (seconds) for each part of an interrupted decision for participants in each strategy condition - Not Instructed and Instructed.

Why would instruction to rehearse the task resumption point have a detrimental effect on performance? One interpretation of these results is that that the instructed participants simply did not use the rehearsal strategy at all. However, instructed participants did have significantly longer interruption lags than participants who were not instructed (see Figure 2, instructed $M=2.80, S D=0.82$ and not instructed $M=2.07, S D=0.73, t(22)=-2.297, p=0.032)$. This indicates that instructed participants were doing something different than participants who were not instructed.

Perhaps participants were not using the strategy as trained? A review of participants' self-reports suggest that participants who were not instructed essentially started over after an interruption (9 out of 11 available reports); they reported doing nothing during the interruption lag to improve their memories for task resumption. In contrast, a majority of participants who were instructed to rehearse the task resumption point did do something to encourage retrieval of the interrupted task information. While only 2 participants reported rehearsing as instructed, 4 participants said that they used some body part, usually their fingers or their hands, to remember the interrupted task information. Four others reported starting over as the non-instructed participants had. Although most of the participants did not use the rehearse strategy as trained, several participants appear to have selected an alternative strategy - the creation of physical memory aids - in lieu of rehearsal. This was a highly adaptive approach and should have been successful. This makes it puzzling that instructed participants performed worse by taking longer to make decisions than participants who were not instructed.

Why might instruction have been detrimental to performance? A few participants reported that they thought it was more important to rehearse the information contained in the message than to rehearse the task resumption point. The message information would be lost after the message left the screen unless they rehearsed it. However, the interrupted task data was available on-screen after the interruption. Instructed participants may indeed have tried to rehearse the task resumption point (or create some physical reminder). However, they valued remembering the message more; the cost associate with forgetting message data was greater than the cost of forgetting the resumption point. But why did strategy instruction result in increased decision-making time on interrupted tasks? This is likely due to interference between memory for the message data and memory for the resumption point.

\section{DISCUSSION}

Analyses suggests that interruptions were costly to decision-makers. On average, 6 seconds, nearly $29 \%$ of the time spent making a decision, was lost when participants were interrupted. Instruction to use the interruption lag to rehearse the task resumption point did not help and may have hurt. Instructed participants seem to have actually taken longer to make decisions when interrupted. This unexpected result is exciting because this is the first task of its kind to integrate investigations of task resumption following an interruption where interruptions are an integral part of overall performance. Therefore, it was possible to see how people make tradeoffs between the memory requirements associated with each. Additional studies have manipulated the cost of forgetting the task resumption point and the costs of forgetting the interrupting message data. The results are currently being analyzed to investigate these memory trade-offs in more detail.

\section{ACKNOWLEDGEMENTS}

This work was supported by grant N0001400WX21058 to Greg Trafton from the Office of Naval Research and by grant F49620-97-1-0353 from the Air Force Office of Scientific Research. Thanks to M. J. Schoelles who developed the I-ARGUS system, W. T. Fu who developed this version of the N-back task, and D. A. Boehm-Davis, J. G. Trafton, E. M. Altmann, 
L. Adelman, R. Holt, C. Monk, and M. Diez for their comments on this research.

\section{REFERENCES}

Altmann, E. M. \& Trafton, J. G. (2002). Memory for goals: An activation-based model. Cognitive Science, 26, 39-83.

Cellier, J., \& Eyrolle, H. (1992). Interference between switched tasks. Ergonomics, 35, 25-36.

Cutrell, E. B., Czerwinski, M., \& Horvitz, E. (2000). Effects of instant messaging interruptions on computing tasks. In Extended Abstracts of CHI 2000, Human Factors in Computing Systems.

Dismukes, K., Grant, Y., \& Sumwalt, R. (1998). Cockpit interruptions and distractions. ASRS Directline Magazine.

Gillie, T. \& Broadbent, D. E. (1989). What makes interruptions disruptive? A study of length, similarity, and complexity. Psychological Research, 50, 243-250.

Kirmeyer, S. L. (1988). Coping with competing demands: Interruption and the Type A pattern. Journal of Applied Psychology, 73, 621-629.
Latorella, K. A. (1996). Investigating interruptions: An example from the flightdeck. Proceedings of the 44th Annual Meeting of the Human Factors and Ergonomics Society.

McFarlane, D. C. (1997). Interruptions of People in Human Computer Interaction: A General Unifying Definition of Human Computer Interruption and Taxonomy. Washington, D.C., Naval Research Laboratory.

O'Conaill, B., \& Frohlich, D. (1995). Timespace in the workplace: Dealing with interruptions. In the Proceedings of CHI ' 95, Conference on Human Factors in Computer Systems.

Schoelles, M. J., \& Gray, W. D. (2001). Argus: A suite of tools for research in complex cognition. Behavior Research Methods, Instruments, \& Computers, 33, 130-140.

Zijlstra, F. R. H., Row, R. A., Leonora, A. B., \& Krediet, I. (1999). Temporal factors in mental work: Effects of interrupted activities. Journal of Occupational and Organizational Psychology, 72, 163-185. 